\title{
El desempeño de los docentes novatos: los practicantes de la modalidad Ce.R.P. en la ciudad de Rivera
}

Karina Nossar*

\begin{abstract}
Resumen
En el presente artículo se exponen los principales resultados de una investigación en la que se buscaron identificar los elementos caracterizadores de los practicantes en esta modalidad de formación docente innovadora, que difiere de los otros modelos existentes en el Uruguay. Se trata de un centro cuyos estudiantes de profesorado realizan la práctica docente en un liceo de práctica, creado a tales efectos, en el que permanecen durante todo un turno, participando de múltiples actividades además de las clases teóricas de Ciencias de la Educación. Se pretende que la práctica docente no quede reducida al espacio del aula sino que abarque la complejidad del centro educativo. Tres de las conclusiones a las que se arribó tienen que ver con las características del modelo de formación, a saber: la mimesis cegadora, que identifica el comportamiento mimético de los practicantes respecto de sus tutores; la saturación atencional de la que son objeto en virtud de la intensa carga horaria y las múltiples responsabilidades que asumen; y la apertura del aula, por cuanto están habituados al trabajo en equipo y a ser observados por colegas y docentes a diario en sus clases. Finalmente, se describe el discurso interrogativo de los novatos y las distintas formas que éste asume, con la peculiaridad de que se relacionan lingüísticamente con sus estudiantes a través de un formato interrogativo que no necesariamente implica que se estén formulando preguntas.
\end{abstract}

\begin{abstract}
This article provides an overview of the main results of research work aimed at identifying the characteristic elements of the practicing teacher-learner in an innovative model of teacher education that differs from other existing models in Uruguay. It focuses on a centre in which teacher-learners carry their professional practice in a special purpose high school, specifically created for this purpose. Teacher-learners stay during a whole shift, taking part in every activity and at the same time receiving theoretical lessons on Education Sciences. The aim is to expand the scope of the professional practice, which is not limited to the classroom but also includes the complexity of the educational centre. Three of the conclusions are related to the characteristics of the teacher's educational model, which are: blinding mimesis, which identifies the mimetic behavior of the apprentices with respect to their tutors; saturation of attention, in view of the long schedules and multiple responsibilities that they assume, and the opening of the classroom, as they become used to work in teams and to be observed daily by colleagues and professors in their classes. Finally, we describe the interrogative discourse of the novices and the different forms it assumes, with the peculiarity that they are linguistically related with their students through an interrogative format, which does not necessarily imply that they are making questions.
\end{abstract}




\section{I ntroducción}

Esta investigación tuvo como objeto el estudio del desempeño de los practicantes de la modalidad Ce.R.P. en la ciudad de Rivera. Esta enunciación conlleva implícitas dos cuestiones: la primera de ellas radica en el hecho de que al Centro Regional de Profesores del NorteRivera asisten estudiantes de toda la región norte del Uruguay, vale decir, de los Departamentos de Cerro Largo, Artigas, Tacuarembó y Rivera. Ello se debe a que una de las características de esta modalidad de formación de profesores es la regionalidad, sustentada en la base de la descentralización.

La segunda de las cuestiones se sustenta en el entendido de que los estudiantes de profesorado de los Ce.R.P., en su último año de práctica docente, son asimilables a lo que en la bibliografía se entiende por docentes principiantes, noveles o novatos. Esta definición que asumimos como válida, obedece a que la práctica en esta modalidad es un componente esencial, con características peculiares. La más sobresaliente de las mismas es que se realiza en parejas pedagógicas con un grupo a cargo, en centros de práctica e insume media jornada de labor, que incluye las clínicas y las coordinaciones con colegas, tutores y profesores de apoyo. Estudiar el desempeño de los principiantes en esta modalidad apunta a la construcción de conocimiento teórico válido para el contexto en cuestión. No hay antecedentes de estudios en este sentido, más allá de las publicaciones oficiales de la Administración Nacional de Educación Pública, las cuales son muy generales ${ }^{1}$ puesto que obedecen al relevamiento de datos, básicamente cuantitativos, necesarios e imprescindibles para la toma de decisiones, y otros trabajos, muy recientes, que dan cuenta de esta innovación educativa, y son de corte descriptivo-evaluativo, tomando en consideración el impacto de este modelo. Ninguno de los trabajos a los que hemos tenido acceso se centra en el estudio de las prácticas de los principiantes, no obstante aparecen mencionadas en todos ellos como uno de los aspectos centrales de la propuesta.

\section{Características del modelo Ce.R.P.}

Los Centros Regionales de Profesores fueron creados dentro del marco de la llamada Reforma Educativa impulsada por la Administración Nacional de Educación Pública en el período 19952000. Los datos recogidos por el Censo de docentes de 1995 arrojaron porcentajes de titulación de profesores de Educación Media que descendían en el interior del país a un 19,6\%. La situación se agravaba en algunos departamentos y en algunas áreas consideradas como críticas. Dentro de este contexto es que se inauguran en 1997 los Ce.R.P. de Rivera y Salto, en 1998 se agrega Maldonado, en 1999 lo hacen Atlántida y Colonia, completando con Florida en el año 2000. De este modo se logra implementar una red de institutos de formación docente con el mandato institucional de formar profesores para paliar la situación del interior de nuestro país.

Los Ce.R.P. poseen características que los diferencian de las otras modalidades de formación de docentes que coexisten con ellos. Se trata de centros regionales, es decir que abarcan toda la zona de influencia, donde se brinda una formación intensa en régimen de dedicación exclusiva, con jornadas de ocho horas durante tres años, lo que totaliza 4200 horas reloj de clase ${ }^{2}$. Para ello es que se ha implementado un sistema de becas que comprenden alojamiento, alimentación y transporte de modo de facilitar a los alumnos su dedicación al estudio. Las opciones ofrecidas son los profesorados de Lengua y Literatura, Matemática, Inglés ${ }^{3}$, Ciencias Sociales con mención Historia, Geografía o Sociología, Ciencias de la Naturaleza con mención en Física, Química o Biología. Los docentes que se desempeñan como formadores también tienen un régimen de dedicación exclusiva en su mayoría, lo que permite un trabajo personalizado y una mejor atención a las necesidades del estudiantado. Por otra parte, se pretende que los Ce.R.P. se posicionen como enclaves culturales, centros de referencia para la comunidad, con docentes de nivel académico, bibliotecas especializadas y que promuevan actividades culturales, encuentros e intercambios entre docentes de la región.

\section{4 - Universidad ORT Uruguay}


El diseño curricular de los Ce.R.P. está estructurado en tres grandes bloques:

- Área específica, que comprende al menos dos asignaturas por año (dependiendo de la opción elegida). En todos los casos estas asignaturas son integradoras de conocimientos, es decir que no se trata del perfil asignaturista tradicional, sino que comprende contenidos del área estructurados de modo de dar una visión holística. A medida que el estudiante avanza en la carrera, tiene que ir eligiendo primero un área disciplinar y luego una mención de egreso.

- Área de Ciencias de la Educación. El énfasis que se ha puesto en esta área distingue notoriamente a los Ce.R.P. de las otras modalidades de formación docente. En primer lugar por la carga horaria asignada; en segundo lugar por tener el cometido de ser el eje articulador de toda la propuesta; en tercer lugar porque la Didáctica y la Práctica docente surgen a partir de la asignatura "Planificación y Gestión de Centros Educativos", lo que trae aparejado que el futuro docente ingrese al aula recién después de haberse interiorizado del centro educativo en su conjunto y su funcionamiento general. Ello brinda una visión sistémica del aparato educativo, para ubicar la disciplina y su rol dentro del mismo.

- Área instrumental. Se compone de dos asignaturas clave para los docentes del futuro: Inglés e Informática. Se ha entendido que los profesores del SXXI no pueden permanecer ajenos a estos dos códigos fundamentales para actuar en el mundo globalizado, en el cual deberán, en cuanto intelectuales, manejar las fuentes del conocimiento, decodificar revistas técnicas, bibliografía especializada que se publica en inglés, en cuanto "nueva koiné", y moverse por Internet, utilizando todas las potencialidades que la herramienta informática ofrece a los fines educativos.

Otra característica que diferencia a estos centros de las otras modalidades de formación docente existentes radica en la manera en que se lleva a cabo la práctica docente. Para ello se han creado los "Centros de Práctica", tomando lo mejor de la tradición de la formación de maestros de Educación Primaria en el Uruguay, que consiste precisamente en el trabajo en escuelas de práctica, centros de gran prestigio, con docentes experimentados, calificados para el trabajo con maestros practicantes, y se conjuga con las "clínicas médicas", modalidad seguida por la Facultad de Medicina en la formación de los cuadros profesionales, donde a partir de la presentación de "casos", se producen el análisis y la teorización. Los estudiantes toman un grupo a cargo en parejas pedagógicas, reciben el asesoramiento y acompañamiento de un profesor experto que se denomina tutor, las visitas regulares de otros docentes de apoyo, realizan las "clínicas" de Psicología del Aprendizaje y de Investigación Educativa en el propio liceo, analizando en conjunto las distintas situaciones que se presentan en la labor cotidiana. En las otras modalidades de formación docente existe una práctica con grupo a cargo, pero que no conlleva obligaciones del practicante en el liceo, más allá de las inherentes a las horas de clase del grupo en cuestión.

Esta caracterización de la forma en que los aprendices de profesores comienzan a ejercer la docencia es la que nos lleva a considerarlos como novatos. Sus primeros contactos con alumnos a su cargo es lo que los define como tales. La bibliografía especializada tiende a considerar noveles o novatos a los profesores durante un período que oscila entre el primer año de práctica, los dos primeros, o hasta cinco años (Marcelo, 1991;1999). A los efectos de este estudio, consideramos novatos a los principiantes en su primer año de trabajo rentado con grupo a cargo.

Nos interesó indagar sobre el conjunto de elementos que caracterizan al accionar docente de los practicantes. Es a ese conjunto al que denominamos "desempeño". Este desempeño está ligado a la enseñanza por cuanto se trata de lo observable en términos de la presente investigación. Podremos buscar si esta enseñanza está enfocada a los aprendizajes de los alumnos, pero no podremos efectivamente constatar si los aprendizajes tuvieron lugar ni el carácter de los mismos, es decir, si se trata de conocimiento frágil o de conocimiento genuino. 
Sin duda que podremos indagar sobre las percepciones que los alumnos tienen con respecto a la enseñanza de sus docentes y a sus propios aprendizajes, sin ligarlos con un sesgo causalista. Nuestro enfoque se centra en las características de la enseñanza de los docentes principiantes y en sus concepciones implícitas o explícitas de la misma. Se partió de un "Perfil del novato" elaborado por el equipo docente, en función de un listado de los elementos sobre los cuales se pretende trabajar en la práctica de tercer año. Ese conjunto de elementos que tienen que ver con la labor dentro y fuera del aula, con lo institucional, lo administrativo y lo actitudinal, compone lo que a los fines de este trabajo denominamos desempeño.

\section{El liceo $N^{\circ} 4$ de Rivera}

Para el Centro Regional de Profesores de Rivera, desde el año 1999, se ha designado el Liceo $N^{\circ} 4$ como centro de práctica. Este liceo posee algunas características que lo hacen especial, aparte del hecho que consignamos aquí. Es un liceo que se construyó en una zona periférica, con escasa población, lo que lleva a que cuente con poca cantidad de estudiantes para la infraestructura que posee. Por este motivo es que funciona en un solo turno, matutino, y exclusivamente con Ciclo Básico de Educación Media, lo que se traduce en cuatro grupos de primer año, tres de segundo y tres de tercero. ${ }^{4}$ El número de alumnos se ha incrementado desde que funciona como centro de práctica, puesto que la ANEP instrumentó un servicio de transporte gratuito desde otros barrios alejados ${ }^{5}$ para que los jóvenes pudieran asistir. Esta medida ha elevado la matrícula a algo más de 200 estudiantes, no obstante, se trata de una población muy especial que proviene de otros barrios periféricos, sin acceso a otros centros educativos por razones económicas y cuyas familias no mantienen relacionamiento fluido con el liceo.

Los docentes practicantes, cuyo número es alrededor de 80 por año, dependen del Director del liceo, pero coexisten en ese centro 4 tutores, 8 profesores de apoyo ${ }^{6}$ y 2 profesores que tienen a su cargo las clínicas, los cuales dependen de la dirección del Ce.R.P. Se trata, por tanto, de una institución muy compleja, que plantea una serie de desafíos al equipo directivo, que todos los años cambia su personal en un 77\% (los practicantes que egresan) sin contar con la movilidad natural que puede registrarse en el resto de los docentes. Por otra parte, es un centro cuya población lleva a que exista una peculiar proporción de dos alumnos por docente aproximadamente, lo que podría pensarse como una condición favorecedora del trabajo interno.

\section{Recorrido teórico}

Luego de realizar la descripción situacional (Sautu, 2003) es decir, definir el contexto sociohistórico en el que el suceso tiene lugar, encuadramos esta innovación dentro del contexto latinoamericano, la política educativa y las reformas que tuvieron lugar. Nos centramos en el análisis de la innovación dentro del sistema, prestando especial atención al hecho de que el pensamiento que ha determinado políticas en el ámbito de la formación docente se podría enunciar como "empecemos por formar los nuevos profesores, que cuando lleguen a las escuelas van a cambiar la enseñanza”. Este argumento ha quedado superado por la realidad, en tanto desconoce las intermediaciones del sistema y el poder de la organización. La literatura sobre el tema nos indica que cuando los nuevos profesores llegan a la escuela no la cambian; más bien ocurre lo contrario: la escuela los cambia a ellos haciendo inútil todo esfuerzo anterior. 
Por otra parte, ya se ha estudiado que el "aprendizaje de la observación" (Lortie, 1975) influye en las prácticas de los novatos. A la hora de enfrentarnos a la tarea de enseñar, ya hemos estado expuestos a miles de horas de observación de docentes desde nuestro rol de alumnos. Estos aprendizajes tienden a aparecer recurrentemente en nuestras prácticas, a pesar de la formación recibida. Muchas veces estas prácticas no se corresponden con las adecuadas para nuestros alumnos porque pese a haber estado expuestos durante tantos años al accionar docente, no hemos tenido acceso a las razones por las cuales los docentes han tomado sus decisiones. Hemos tenido acceso a un conocimiento docente de superficie, el cual se dio en un tiempo y en un lugar determinados, lo que lo convierte en poco extrapolable a otros contextos.

El sistema educativo es un todo complejo y es poco posible cambiarlo si no se lo encara con un enfoque sistémico (Tedesco, 1998). Los esfuerzos por cambiar la formación docente independientes de todo otro esfuerzo de cambio en otras partes del sistema, no han resultado, por tanto, exitosos. En el espíritu de la reforma educativa impulsada por Rama (período 19952000) estaba presente esta idea, de allí que se pretendió actuar en todos los niveles del sistema educativo. También es cierto lo señalado por Aguerrondo (2002) con respecto al "timing" de los cambios. La fuerza del cambio disminuye con el paso del tiempo, esto quiere decir que en el momento en que tiene más fuerza, abarca la parte más pequeña de la organización. A medida que pasan los años la fuerza disminuye, pero los patrones tradicionales de funcionamiento se mantienen vigentes y, aunque se siga avanzando en la aplicación, es probable que la innovación se "burocratice", es decir que los cambios se produzcan formalmente pero en realidad, se siga trabajando igual que antes, aunque sea con las nuevas denominaciones.

Por otra parte, relevamos la bibliografía sobre teorías de los ciclos vitales, concentrándonos en la primera etapa en la vida profesional de los docentes, lo que nos llevó al terreno de los principiantes, especialmente a la caracterización de Marcelo (1999) y la descripción de la situación en la que se incorporan a sus puestos de trabajo. Se destaca como característica la inseguridad y la falta de confianza en sí mismos que padecen los profesores principiantes. El primer año, (el que nos interesa) es en general, un proceso de intenso aprendizaje, del tipo ensayo-error, marcado por un principio de supervivencia, y por un predominio del valor de lo práctico. Los profesores principiantes se encuentran con ciertos problemas específicos de su estatus profesional: la imitación acrítica de conductas observadas en otros profesores, el aislamiento de sus compañeros, la dificultad para transferir el conocimiento adquirido en su etapa de formación, y el desarrollo de una concepción técnica de la enseñanza. Marcelo analiza las realidades en otros contextos, encontrando un auge de los llamados programas de iniciación.

Los mismos se entienden como extensiones lógicas del programa de formación inicial, y como piezas de entrada en un programa más amplio de carrera docente. Estos programas se basan en el reconocimiento de que los principiantes recién han culminado su período de formación y aún necesitan supervisión y apoyo similares a los que recibieron siendo estudiantes. Los programas de iniciación se configuran, por tanto, como el eslabón imprescindible que ha de unir la formación inicial con el desarrollo profesional a lo largo de la carrera docente y tratan de establecer estrategias para reducir o reconducir el denominado "shock de realidad"7 . La fundamentación y los objetivos de dichos programas son fácilmente extrapolables a nuestra realidad educativa, dado que en el Uruguay es tradición que la puerta de ingreso al mercado laboral en la docencia sea la que más dificultades presenta. Las distintas figuras que aparecen en la bibliografía como protagonistas en este rol de apoyo a novatos, llámense asesor, mentor, monitor, tutor, etc., comparten en esencia la responsabilidad de acompañar al principiante en esta etapa, brindándole los elementos ausentes en la formación inicial, pero fundamentalmente, promoviendo el bienestar personal y profesional para minimizar los efectos del "shock de realidad". 
El principal objetivo del proceso de asesoramiento es realizar una transformación, un cambio desde un estado de imitación al de autogestión.

Nuestro recorrido teórico pasó también por el análisis de la conceptualización de la profesión y el profesionalismo docente, las competencias de un profesor, el estudio del perfil del novato, para finalmente desviarse hacia temáticas de carácter lingüístico que aparecieron como consecuencia del estudio de la interacción en el aula. En este sentido, aflora naturalmente el análisis de la situación especial de la frontera con Brasil, dadas las interferencias del portugués en las aulas como reflejo de esta sociedad bilingüe y diglósica ${ }^{8}$.

\section{Decisiones metodológicas}

El universo a investigar constó de 61 docentes novatos que realizaron su práctica en el año lectivo 2004 en el liceo $N^{\circ} 4$ de la ciudad de Rivera. En virtud del número elevado a efectos de una investigación, se decidió acotar la muestra a razón de cuatro practicantes por área. En ese sentido se les solicitó a los docentes tutores que sugirieran nombres de novatos que ellos consideraran como los más representativos de la generación. Se pidió expresamente que no fuesen extraordinarios en ningún sentido, y que su perfil fuese acorde con la media de los practicantes de esta modalidad desde la primera generación hasta el presente. De esta forma se definieron los 16 sujetos con los que se emprendió esta tarea.

Las clases fueron grabadas y observadas, con la pauta de una planilla. A posteriori se realizaron entrevistas al novato, al tutor y/o profesor de apoyo y al director del liceo a partir de un cuestionario-guía. En lo que respecta a los alumnos de los practicantes, se les aplicó una encuesta anónima por medio de la opción de "falso-verdadero". A efectos de no generar condicionamientos, se esperó a que los novatos rindieran su examen final de práctica docente y se le pidió al tutor, actor con el que los adolescentes están familiarizados, que realizara la tarea en ausencia del novato.

Por otra parte, se incorporaron las planificaciones de las clases observadas, como un documento más a efectos de contrastarlo con lo efectivamente ocurrido y formular preguntas en la entrevista basándose en lo que fue pensado de antemano y su respectiva justificación.

\section{El camino de los datos}

En una primera instancia, se fijaron una serie de aspectos que se consideraron dignos de ser observados, buscando caracterizar a partir de ellos, las prácticas de los novatos. A medida que fue avanzando el trabajo de campo, los propios datos, en el proceso de lectura y relectura en la búsqueda de recurrencias, fueron indicando el camino a seguir. Nuestro interés se fue centrando, de esa manera, en la transcripción de las clases y en particular en la interacción a partir de las preguntas que los novatos formulan en las mismas. Por otra parte, encontramos que los principiantes pertenecientes a una misma área poseían rasgos comunes. Recordemos que para cada una de estas áreas existe un tutor diferente, lo que puede ayudarnos a aventurar la búsqueda de una posible explicación que tuviera que ver con la marca del modelo docente. En un primer nivel de análisis nos focalizamos en las distintas áreas, sus similitudes y diferencias, para luego pasar al segundo nivel e interpretar lo obtenido.

Los elementos que en principio se señalaron para dirigir la mirada en las clases se fueron agrupando en dimensiones de análisis, a saber:

- Manejo de grupo

- Motivación, uso de recursos y creatividad

- Planificación, secuencia y contenidos

\section{8 - Universidad ORT Uruguay}


- Compromiso con el centro y cuestiones administrativas

- Capacidad de trabajo en equipo y receptividad a críticas y sugerencias

- Desplazamiento por el aula, interacción e interrogación.

En todos estos ítemes hemos encontrado recurrencias, tanto en la observación de clases como en las entrevistas. Solamente de la última dimensión podemos decir que lo hallado es generalizable a todos los novatos, independientemente del área a la que pertenecen.

\section{La mimesis cegadora}

A partir de la evidencia que significó el vernos obligados a analizar por separado cada una de las cuatro áreas de acuerdo con las diferencias y similitudes existentes, es que podemos concluir que en el modelo Ce.R.P. quedaría al descubierto la marca de los modelos docentes a modo de "mimesis cegadora". No es novedoso para nada el afirmar que los modelos docentes vivenciados, especialmente en la etapa de formación, son los que se internalizan y reproducen en esta etapa de la vida profesional, o bien porque desde su rol de alumnos asumen que mimetizarse implica satisfacer a los tutores, o bien porque se sienten identificados positivamente con sus modelos.

Tomemos como ejemplo la disciplina, tema que importa a los novatos -pero no tanto como se esperaba-, en función de que a nivel internacional es una de las dos grandes preocupaciones junto con el dominio del saber. Sólo dos fueron capaces de identificar el correlato con la motivación, para el resto es una suerte de prerrequisito para trabajar y debería estar dada de antemano. No ven su directa responsabilidad en el tema, dado que cuando se generan problemas, la culpa está siempre en otro lado, generalmente en los alumnos, o bien porque no estudian, o porque son difíciles, o porque son repetidores, o a causa del horario de las clases, o por la disposición del salón o incluso por tratarse de un liceo de práctica. Pero lo más notorio es que si el tutor no observa el problema o no le adjudica demasiada importancia, el novato se hace eco de la misma postura. Existe una visión compartida con el modelo docente de quien los guía, al tiempo que, desde su rol de alumnos, se ocupan de aquellas cuestiones que el profesor experto les señala. Esta esquizofrenia de ser al mismo tiempo profesores y alumnos en un mismo centro, no siempre es resuelta en beneficio de los estudiantes de Ciclo Básico.

Adjetivamos este comportamiento mimético señalando la "ceguera" del novato en la medida en que no cuestionan ni ven más allá de lo que el tutor les indica. Al mismo tiempo, si el tutor no acompaña sus observaciones con el debido peso en las evaluaciones, los practicantes perciben el doble discurso y ponen el énfasis en aquellos aspectos que saben que serán los fundamentales desde la visión del tutor, aspectos que forman parte de la "evaluación oculta" que todos conocen sin que jamás se explicite dado que forma parte de lo "políticamente correcto" en el discurso docente. Nos referimos con ello a cuestiones como el compromiso con el centro educativo, del que unánimemente se habla porque ha invadido todos los ámbitos académicos, pero al que, en última instancia, se le asigna un peso simbólico.

Paralelamente a la "mimesis cegadora" de la que son víctimas los novatos, se puede observar que los tutores, por la fuerte convivencia que genera este modelo, desarrollan una subjetividad que nubla la visión de lo que efectivamente ocurre en las clases. De esta forma es que nos hemos encontrado con tutores que afirman que no existen problemas de dominio de grupo en novatos cuyas clases analizamos, y en las que detectamos serias dificultades en este sentido. 
Es decir que, como complemento de la mimesis del practicante, se produce una ceguera del tutor que le impide ver las debilidades de sus alumnos, forjados a su imagen y semejanza. Esta simbiosis en la que se desdibujan los roles de unos y otros, crece a medida que los lazos afectivos son más fuertes. Situación análoga ocurre en lo que tiene que ver con las exigencias de la planificación, la secuencia didáctica planteada y el grado de dominio de los contenidos: el principiante se adecua desde su rol de alumno a las directivas que le son impartidas.

En cuanto a la motivación, el uso de recursos y la creatividad, su agrupamiento responde básicamente al hecho de que los entrevistados suelen asociarlas. Cuando se les pregunta por la forma en que motivan a los alumnos, inmediatamente se correlaciona la motivación con el uso de recursos y por consiguiente, con la creatividad que permite contar con un mayor abanico de posibilidades. No obstante, siguen existiendo diferencias entre las distintas áreas en cuanto a las concepciones que manejan al respecto: los novatos se apropian del discurso y la visión del tutor. Si bien persisten las diferencias entre las cuatro áreas, se encuentran mayores coincidencias entre Ciencias Sociales y Lengua y Literatura por una parte, y por otra, Matemática y Ciencias de la Naturaleza, en lo que tiene que ver con esta temática. Los recursos en las dos últimas áreas mencionadas responden a la necesidad de visualizar conceptos abstractos o de atender a los estilos de aprendizaje, mientras que en las otras dos áreas ha costado más su utilización adecuada y por momentos resultan decorativos o incluso se fuerzan la clase y la planificación en función del recurso seleccionado. En términos generales, se observa que cuando se les plantea el tema de los mediadores de aprendizaje, piensan en primera instancia en herramientas sofisticadas para el trabajo en el aula, que requieren tecnología.

La mimesis cegadora se conjuga con el peso del "aprendizaje de la observación" del que habla Lortie, el cual subyace a nivel subconsciente o inconsciente, y aflora especialmente en situaciones de altas presiones. Se da entonces una suerte de regresión en la medida en que los esfuerzos de la institución formadora parecen vanos al enfrentarse a toda una vida de sometimiento a otros modelos docentes que han internalizado. Más allá de que la formación docente pueda ser concebida como una empresa de "bajo impacto" (Terhart, 1987), aquí no se trata del centro en su conjunto con sus metas y proyectos, sino de la modelización a cargo de un tutor, quien a su vez tiene el poder de la calificación en sus manos.

El cumplimiento de las tareas administrativas por parte de los novatos no ofrece reparos en términos generales en virtud de su doble rol en el centro educativo. En este sentido, consideran que las reuniones de coordinación son de corte administrativo, y asisten a ellas tomándolas como una obligación más. La mayoría entiende que calificar a sus alumnos, realizar prueba diagnóstica, planificar y tomar medidas disciplinarias cuando corresponde, son tareas administrativas. Nos encontramos aquí frente a un tema semántico porque no se les explicitó en las entrevistas a qué tareas concretas nos referíamos al preguntar por "cuestiones administrativas de la labor docente", precisamente porque queríamos sondear qué cosas caían dentro de la dimensión administrativa desde la percepción de los distintos actores de la comunidad educativa. Se puede deducir al respecto, que los novatos perciben como labor administrativa todo aquello que implica trabajar con documentos escritos, principalmente cuando son a requerimiento de la dirección del centro. El asistir a reuniones de coordinación es tomado como cuestión administrativa en la medida en que los novatos declaran que efectivamente, las reuniones de coordinación tienen ese carácter. De allí parten también sus resistencias y sus reparos, lo que va acompasado de un relacionamiento conflictivo con la dirección del centro. 
El tema del doble rol de los practicantes en el liceo 4 tiene su contrapartida en los docentes tutores o de apoyo a la práctica por un lado, y en el equipo de dirección del liceo por otro. También ellos sufren de esta esquizofrenia que no les permite tener clara su función y los límites de la misma. Estos "difusos límites del rol" en el centro asumen distintas manifestaciones que terminan siempre en relegar zonas de posibles conflictos. Es así que la colaboración con la institución y el compromiso con la misma no es un área en la que los tutores trabajen especialmente. Por su parte, los docentes del Ce.R.P. tampoco están cumpliendo sus funciones dentro del local de la institución a la que pertenecen y se sienten extranjeros en el liceo. Al no desarrollar el sentido de pertenencia a ese centro, no pueden transmitirlo a sus alumnos practicantes. Si bien reconocen su importancia, no lo toman en cuenta de la misma forma a la hora de evaluarlos. A su vez deberíamos agregar el hecho de que existen vacíos legales puesto que se carece de normativa clara a todos los efectos que implica el funcionamiento de un liceo de práctica.

El compromiso con el centro educativo es, en todos los casos, algo a rever y repensar desde la institución formadora, en la medida en que los estudiantes están siendo moldeados desde la lógica de la legitimidad del doble discurso.

\section{Saturación atencional}

Los principiantes se encuentran bajo una fuerte presión que implica el tener que atender varios frentes a la vez. Consideramos que ello se produce no sólo por el hecho de estar iniciándose en la docencia, sino también por el régimen de dedicación exclusiva de este modelo de formación inicial. Por otra parte, vale agregar que desde sus inicios, el proyecto Ce.R.P., marcado por el principio de equidad social, pretende reclutar alumnos que por su situación económica no pudiesen cursar su carrera en la capital. La situación económica viene de la mano con una realidad sociocultural deprimida, lo que acarrea en los hechos una matrícula con un "back-up" muy distante del perfil de ingreso a la docencia en el I.P.A9 . Más allá de nuestra convicción idealista, los estudiantes necesitan determinadas bases a partir de las cuales comenzar a delinear un futuro docente. Las más de las veces carecen de ellas, por lo que se ven obligados a reduplicar esfuerzos y así subsanar las inequidades resultantes del pueblo de origen, el hogar o el bachillerato que pudieron cursar, dado que no se ofrecen en todos los liceos las mismas opciones.

Abordaremos la explicación de aquello a lo que denominamos "saturación atencional" desde los aportes de la ciencia cognitiva, en especial mediante la clasificación en tipos de conocimiento (Aparicio, 1995). El conocimiento declarativo, que se compone del episódico y del genérico es descriptivo y consciente mientras que el conocimiento procedimental, que es el más importante, no es consciente. Este último es el que nos sirve para hacer cosas y debe estar automatizado. Cuanto más compleja es una tarea y por ende, el procedimiento, más automatizada debe estar. El conocimiento declarativo debe estar al servicio del procedimental, puesto que en sí mismo es inerte.

Ahora bien, el volumen de conocimiento declarativo que los estudiantes deben almacenar en tan sólo tres años de formación en un régimen tan intenso como el que nos ocupa, hace imposible que sea traducido a procedimental y en consecuencia, automatizado. Debemos considerar además, que el diseño curricular original de los Ce.R.P. parte del conocimiento teórico en los primeros años y recién hacia el final de la carrera se produce la entrada al aula. Es decir que reciben el conocimiento declarativo que mucho más adelante necesitarán para convertir en procedimental. Se les inhibe de esta forma de descubrir por sí mismos la necesidad de contar con él y buscarlo motivados por el deseo. 
Nuestros novatos no han contado con los tiempos de maduración y reflexión que se requieren para ir traduciendo sus conocimientos declarativos a procedimientos automatizados y se ven desbordados frente a las exigencias cotidianas de forma que su atención se dispersa en múltiples aspectos inabordables. Por tanto, y utilizando una metáfora que proviene del campo informático, entran en "overflow". Esta situación se produce cuando una computadora recibe más órdenes de las que es capaz de procesar de acuerdo con su configuración, y el administrador de tareas bloquea unas cuantas de ellas. De forma análoga, el novato que padece de saturación atencional, entra en "overflow" por sobrecarga, y sólo es capaz de atender algunas cuestiones relegando el resto o simplemente, ignorándolo. Como ya hemos argumentado, la selección de prioridades estará condicionada por la visión del tutor, administrador de tareas en la práctica docente. Se conjugan así, la mimesis cegadora y la saturación atencional.

Como mecanismo de supervivencia frente a esta saturación de la que son objeto, generan procedimientos basados en concepciones alternativas, las que como hemos visto, provienen del "aprendizaje de la observación". Estos conocimientos resistentes están automatizados y encajan en la ecología conceptual del sujeto, se conectan a las creencias y explicaciones, configurando una suerte de paradigma explicativo. Un primer paso para revertir esta situación tendría que pasar por tomar conciencia de ello al tiempo que nos debe mover un deseo de crecimiento profesional. Dicho en otros términos, para que se produzca un cambio conceptual, resultan esenciales la metacognición y la motivación. No podemos pensar en el conflicto ni la analogía como mecanismos, sin un sujeto consciente de sus procesos cognitivos y fuertemente motivado por el conocimiento. Si admitimos que el sentido último del conocimiento está en el uso que se hace del mismo, estamos admitiendo que el sujeto aprendiz se motivará al vislumbrar su utilidad. Este proceso es lento y tiene tiempos que deben ser respetados de modo que cada individuo logre automatizar algunos aspectos de su práctica basado en un conocimiento declarativo correcto para cada situación, lo que favorecerá el pasaje del estado de imitación al de autogestión.

\section{La apertura del aula}

Otras investigaciones (Mancebo, 2003; Cifra, 2003) han dado cuenta de la forma en que los egresados de los Ce.R.P. se comportan en el momento de incorporarse a otros centros educativos como docentes. Ha sido ésta una de las marcas identificatorias de quienes provienen de esta casa de estudios: su facilidad para trabajar en equipo, su buena disposición y apertura. La modalidad de formación adoptada desde la concepción del diseño curricular de los Ce.R.P. prevé que la práctica docente se inicie en segundo año desde la mirada al centro educativo con la asignatura Planificación y Gestión de Centros, a partir de la cual, y en coordinación con el profesor de Didáctica, los estudiantes ingresan al aula. Tanto en la práctica de segundo como en la de tercero, lo hacen en parejas pedagógicas, compartiendo la responsabilidad, planificando, estudiando y evaluando en conjunto. Esta entrada al aula se acompaña con una fuerte presencia de docentes experientes, que se encargan de realizar varias visitas a las clases, las que a su vez, son en conjunto con los profesores de apoyo. Paralela y simultáneamente, los docentes que tienen a su cargo las clínicas de Investigación Educativa aplicada y Psicología de la Educación, las que se desarrollan en el centro de práctica, también ingresan al aula para observar clases de practicantes y partir de lo efectivamente ocurrido para abordarlo a nivel teórico. Los compañeros de la misma área y los de otras áreas suelen observar a sus pares y compartir con ellos el análisis de las clases. Varios docentes del Ce.R.P. y la Dirección del mismo visitan periódicamente a los practicantes realizando a posteriori las devoluciones correspondientes. 
Todas estas miradas que confluyen en las prácticas las enriquecen y generan una actitud de diálogo permanente basado en críticas y sugerencias varias, de tal suerte que los principiantes han internalizado el hecho de no estar solos con sus alumnos como la práctica más habitual. Todos valoran el trabajo en parejas como muy positivo y reciben las múltiples visitas como una rutina más dentro de una absoluta normalidad. Esta forma de encarar la práctica les proporciona un entrenamiento que permite abrir las puertas del aula y quedar expuestos a las miradas de otros sin que ello les genere ansiedades y temores propios de quienes nos formamos a puertas cerradas. Es por este motivo que consideramos que uno de los efectos de la formación en este modelo, que se evalúa como fortaleza, es la posibilidad de trabajo en equipo, crítico, reflexivo, que se puede potenciar en la medida en que continúen en esta línea al egresar e insertarse laboralmente en otros centros educativos. Ya hemos señalado el poder neutralizador del sistema y confiamos en que los equipos directivos perciban el enorme potencial a desarrollar existente en estos novatos de tal forma que no se desperdicie mediante la burocratización y rutinización de las prácticas.

En lo que respecta al desplazamiento por el aula, además de lo que se desprende de las entrevistas, contamos con el croquis del salón que se fue realizando en cada una de las clases observadas. Allí se señaló el movimiento del practicante por el espacio físico, encontrándose que no todos hacen un uso adecuado del mismo. Varios de ellos quedan acorralados contra el pizarrón, en una suerte de línea defensiva, sin atreverse a acercarse a los alumnos. Lo corporal, en cuanto a la cinesia y proxemia, deja de manifiesto el temor subconsciente del inexperto. Se puede establecer así un correlato entre la forma de tratamiento lingüístico elegida (tú, vos, usted) entre los pronombres personales disponibles en el sistema, y el desplazamiento por el aula. Aquellos practicantes que eligen marcar distancia desde lo lingüístico, son precisamente los que únicamente se desplazan en la primera línea, es decir, entre el pizarrón, el escritorio, donde por cierto se encuentra la libreta, elemento de coacción, y la primera fila donde se sientan precisamente los alumnos "modelo", los que estudian, participan y son más respetuosos de la autoridad docente. Se produce entonces, una barra de contención, que le brinda al novato la seguridad territorial. Esta "área docente protegida" de la que únicamente el profesor, dueño del saber es usuario, propietario y señor, queda más marcada en las clases donde encontramos problemas de disciplina. Cuando un alumno es invitado a trasponer ese umbral, es para escribir algo en el pizarrón, o señalar en un mapa o en un cartel; de este modo, y por unos breves instantes, se apodera de una pequeña parcela del saber, que el docente generosamente cede desde su buen entender, recuperándola en forma inmediata.

\section{El discurso interrogativo: forma y contenido}

\section{a) El interjuego discursivo}

La relación afectiva que une docente-alumnos tiene en este contexto otro efecto: ambos se encuentran en una situación similar, ambos comparten el hecho de ser estudiantes y de estar siendo evaluados por un superior en la cadena jerárquica, lo que conlleva la comprensión y solidaridad. Se producen así varias secuencias de habla en las que es notorio que el alumno desea ayudar a su profesor, máxime cuando percibe que el mismo tiene dificultades para preguntar o expresarse. Lingüísticamente, podríamos hablar aquí de un "interjuego discursivo", especie de texto construido entre los interlocutores, en el cual la interrogación tradicional asume un formato particular similar al "fill in the blanks". De este modo, el docente inicia un enunciado que es completado por el o los alumnos; al mismo tiempo, el docente se identifica con la posición del alumno, colaborando con él en forma solidaria y lo ayuda a completar sus ideas. Se produce así el "interjuego discursivo" en ambos sentidos, en muchas oportunidades el interjuego abarca más interlocutores, de tal suerte que uno inicia la frase, la continúa el segundo y la finaliza el tercero, o hablan varios a coro. 


\section{b) La sordera del novato}

El modus operandi típico de los practicantes en sus interacciones lingüísticas combina el interjuego discursivo con lo que dimos en llamar "sordera del novato". El diálogo en el aula se identifica por la presencia del modelo IRE (iniciación, respuesta, evaluación), como estructura no marcada, es decir como la estructura común y esperable. En el caso de los novatos, hemos identificado que el modelo IRE, si bien está presente, asume un formato que se distancia de lo que se esperaba, dando lugar a lo que denominamos "sordera". En el modelo de referencia, las secuencias se producen fluidamente a lo largo de las clases; el docente formula la pregunta, el estudiante la responde y el docente retoma a su turno la evaluación que corresponda, sea estimulando positivamente la respuesta, ampliándola, reformulándola o negándola por incorrecta, dando las explicaciones del caso.

Los novatos de nuestra muestra adaptan el modelo IRE a las respuestas preconfiguradas, vale decir que desde el momento en que planificaron el desarrollo de la clase, previeron las respuestas que esperaban a sus preguntas, pero cuando las mismas no se dan, su inexperiencia los lleva a perseguirlas de formas diversas categorizables en las siguientes modalidades:

- Insistir repitiendo textualmente la pregunta

- Reformular la pregunta basándose en la respuesta obtenida

- Ignorar la respuesta incorrecta

- Escuchar la respuesta esperada aún cuando no sea dicha

- Completar la respuesta del alumno

En cualquiera de estos casos, la tónica común es la "sordera", no por necedad sino por la falta de experiencia, flexibilidad y conocimientos que permiten al experto manejar estas situaciones. Como ya hemos dicho anteriormente, las exigencias que pesan sobre el novato son tantas y tan diversas, que no logra atender todos los frentes a la vez. Por ello es que, cuando planifican las clases les cuesta salirse del guión preestablecido, atender a los emergentes, improvisar, y sobre todo estar alertas a respuestas que los lleven a sitios no previstos que les generan inseguridad.

\section{c) El silencio tan temido}

Conjuntamente con esta "sordera" se produce otro fenómeno más que tiene que ver con el formato interrogativo, se trata de una suerte de bombardeo de preguntas, muchas de las cuales no esperan respuesta, no por ser preguntas retóricas, sino porque la propia inseguridad lleva a evitar los silencios en el aula. Cuando el practicante no obtiene la respuesta deseada, o se da cuenta de que formuló mal la pregunta, sigue con una secuencia de preguntas concatenadas que no dan espacio para la intervención del alumno. Incluso dentro de ese monólogo que protagoniza el docente, se suceden las preguntas y sus correspondientes respuestas sin que exista el menor silencio entre ambas.

\section{d) Nunca digas no}

En el modelo IRE del novato, se evita por todos los medios tomar la respuesta incorrecta del alumno, ya sea para analizarla, para trabajar sobre el error o simplemente para indicar que la misma está mal. El novato percibe como agresión el hecho de decir "no", por lo que adopta otras estrategias frente a las respuestas no esperadas. Detectamos que en el marco de la no agresión al alumno frente a respuestas incorrectas, el docente elige el modo interrogativo con un tono especial para repetir la referida respuesta. De este modo, al estudiante le queda claro que se trata de un error porque el docente lo condiciona mediante el rasgo suprasegmental ${ }^{10}$ elegido. O bien acepta la respuesta aun cuando no sea la esperada y reformula la pregunta, pero en ningún caso se les dice que no.

\section{4 - Universidad ORT Uruguay}




\section{e) Me hago eco de tus palabras}

Una última observación en cuanto al modelo IRE consiste en que la evaluación se manifiesta a través de la repetición de la respuesta dada por el alumno, una vez que se la consigue a satisfacción del practicante, es decir que se retoma la respuesta del alumno, legitimándola desde la enunciación a cargo del docente. La estructura no marcada del modelo IRE presenta expresiones del tipo muy bien, correcto, excelente, etc. como respuesta evaluativa. El hecho de que el profesor repita exactamente lo dicho por el alumno, adoptando la "estructura en eco", implica que acepta la respuesta como correcta sin necesidad de estímulo añadido para quien la enunció.

Para finalizar con el análisis de la interrogación en el aula y atendiendo al contenido de las mismas, tomamos como base la clasificación de preguntas de Rajadell y Serrat ${ }^{11}$ en:

- Convergentes

- Divergentes

- Evaluativas

- Regulativas

Desde esta clasificación, estudiamos las preguntas de los novatos, encontrando que las preguntas divergentes y las evaluativas aparecen mínimamente, mientras que las convergentes y las regulativas acaparan el discurso interrogativo. Al respecto aclaremos que se adoptó la decisión de incorporar dos tipos de preguntas con una muy alta frecuencia de aparición, que si bien no son estrictamente regulativas, de algún modo cumplen con esa función. Se trata fundamentalmente, por una parte, de preguntas cuasi-retóricas en el sentido de que no esperan respuesta, sino que aseveran algo de lo que se pregunta el acuerdo o recuerdo de los alumnos: ¿se acuerdan, muchachos? ¿están de acuerdo, chiquilines? Es imposible que frente a este tipo de preguntas, los alumnos contesten ni afirmativa ni negativamente, por ello es que consideramos que son cuasi-retóricas, dado que se condiciona la respuesta y se la da por sobreentendida. Habitualmente el condicionamiento de la respuesta se ve reforzado por la curva melódica que el novato le imprime a estas preguntas, además del hecho de que no se proporciona tiempo para que los alumnos piensen y contesten.

Por otra parte, existe gran cantidad de preguntas que en realidad son marcadores conversacionales disfrazados con la máscara de la interrogación. Los marcadores conversacionales son giros lingüísticos que el hablante utiliza para llenar espacios vacíos en su discurso oral. Muchos de ellos logran convertirse en muletillas cuando se repiten insistentemente (digo, o sea, bueno, etc.) Los novatos temen los silencios en el aula, les generan ansiedad y no saben qué hacer con ellos, por lo que los evitan como mecanismo de defensa para evitar quedar expuestos. Precisamente como estrategia de llenado de silencios, y aunado a la concepción implícita de que el docente debe preguntar, adaptan los marcadores conversacionales al modo interrogativo. En ambos casos no se espera respuesta, y dado que su función en última instancia es regulativa, las contabilizamos como tales en el modelo propuesto.

Tomamos entonces como pregunta cada expresión lingüística con formato interrogativo y llevamos a porcentajes lo obtenido. De esta forma, los marcadores conversacionales que adoptan este formato, aparecerán como preguntas regulativas, de acuerdo con el criterio esgrimido, pero las colocamos aparte para dar cuenta de su alta frecuencia. Vale aclarar que, dado que se trata de idiolectos, o sea de hablas individuales, en algunos casos los marcadores conversacionales operan como verdaderas muletillas con una altísima frecuencia de aparición que responde a características personales del hablante y no puede generalizarse como marca identificatoria del discurso de los novatos. Es notorio el hecho de que del 100\% de las preguntas 
efectivamente formuladas en las clases transcriptas, que totalizan 2055 enunciados interrogativos, un $63 \%$ de las mismas corresponden a las de tipo convergente. Recordemos que las preguntas convergentes son aquellas que buscan una respuesta de memorización y se caracterizan por ser cortas y concretas, respondiendo a una enseñanza inductiva. Estas preguntas admiten toda clase de formulaciones y se contabilizan todas sus repeticiones con sus variantes.

Las preguntas divergentes reciben respuestas amplias, inician discusiones y exigen habilidad para escuchar, de las que hemos detectado tan sólo 18, o sea un 0,9\% del total. En muchas oportunidades las preguntas se inician como divergentes, pero se convierten en convergentes por cuanto no se escuchan las respuestas y no se inicia discusión alguna. Las preguntas evaluativas que buscan un juicio de valor por parte del alumno totalizan 24 , que corresponde a un $1,17 \%$.

Finalmente, las de corte regulativo, que organizan y controlan el aula, tienen una presencia de un $16,7 \%$, a las que sumamos las que responden a marcadores conversacionales con un $18,1 \%$, contabilizando un $34,8 \%$ del total. Veámoslo en un gráfico, ya que puede resultar ilustrativo de la interrogación de los novatos:

En términos generales, podemos afirmar que en la interacción lingüística de los novatos existe una importante presencia de preguntas convergentes, siguiéndole en orden de frecuencia de aparición las regulativas, mientras que en ínfima medida se producen las evaluativas y las divergentes.

Este modesto estudio estadístico da cuenta de la necesidad de romper con modelos tradicionales de enseñanza que, a pesar de encontrarnos embarcados en un centro innovador, siguen estando presentes en las aulas de formación docente, influencia inmediata que vivencian los practicantes y que se combina con sus trayectorias educativas anteriores, seguramente también tradicionales.

La pregunta es la estrella en el salón de clase, de hecho, es el único ámbito en el que se formulan preguntas cuyas respuestas se saben de antemano. Los estudios sobre la interacción en el aula, si bien son recientes, revelan resultados que todos intuíamos. Hemos relevado trabajos al respecto y hemos utilizado alguno de ellos para nuestro análisis. Una clasificación más sencilla es la de Burbules (1999) en preguntas aparentes y preguntas auténticas, la cual no quisimos tomar dado que nos interesaba especialmente indagar con mayor profundidad en las llamadas aparentes, las que el propio Burbules defiende por su pertinencia en algunos momentos y con determinados fines. Las preguntas regulativas nos resultaron ilustrativas para seguir la línea de Gabbiani (2000) y sus estudios sobre el lenguaje como instrumento de poder en el aula. En función del conteo que realizamos de las preguntas que aparecen en las clases observadas es que podemos afirmar que los novatos asumen el modo interrogativo para relacionarse lingüísticamente con sus alumnos.

Utilizar el modo interrogativo no significa necesariamente preguntar, vale decir que el que denominamos modo interrogativo es el formato elegido como vedette del discurso. El mismo aparece básicamente en tres situaciones comunes:

- En el control del aula para suavizar órdenes y directivas en enunciados del tipo: ¿te podés sentar?

- En el llenado de vacíos discursivos que tienen la función de mantener el turno del diálogo, o sea, como marcadores conversacionales: ¿verdad? ¿sí? ¿tá? Cumplen esta función también los enunciados del tipo ¿están de acuerdo? puesto que no esperan respuesta ni se proporciona tiempo para la misma.

\section{6 - Universidad ORT Uruguay}


- En la exposición tradicional, para matizar la misma incorporando preguntas cuya respuesta proporciona inmediatamente el mismo docente: una corriente de agua fría, marina, que lo que hace sobre la costa peruana desciende las temperaturas entre 5 y 8 grados, ¿tamos? ¿entonces qué ocurre? Ustedes me dirán, bueno, si no pueden pasar las nubes de humedad cargadas por la cordillera, no importa porque tenemos el sol, tenemos agua suficiente, se evapora el agua, se cumple perfecto el ciclo del agua, igual llovería en las costas, bueno, viene esta señora y les dice, no, no va a pasar, ¿por qué? Porque desciende la temperatura aproximadamente entre 5 y 8 grados va a dificultar la evaporación del agua, el agua está muy fría, no se va a evaporar, si no se evapora, no asciende, no se forman nubes, no llueve. Este tipo de secuencias incorporan el formato interrogativo sin que en realidad se trate de preguntas. Su función a nivel discursivo es la de producir variaciones en la curva de entonación de modo de captar la atención del oyente con un formato melódico apelativo que quiebra la monotonía de la exposición.

Del resto de las preguntas que se formulan, como ya fuera analizado, la inmensa mayoría corresponden a las del tipo convergente o aparente. Sea cual fuere la clasificación adoptada, lo que queda en evidencia es que la pregunta verdadera, la auténtica, la que queda fundamentalmente abierta, cuya respuesta no está establecida y por tanto promueve deseo y curiosidad, ese tipo de pregunta es la gran ausente en las aulas. Lo que hemos visto es una superabundancia de enunciados que adoptan la curva melódica de la interrogación donde la forma y el contenido se divorcian, es decir que la forma es interrogativa mientras que el contenido no apela a una respuesta que exija reflexión, investigación, argumentación y/o discusión.

\section{Notas finales}

"Para que la enseñanza mejore de forma significativa, es preciso crear una tradición de investigación que sea accesible a los docentes y nutra la enseñanza". Este pensamiento de Stenhouse ha alimentado a varias generaciones de docentes e investigadores que buscan compartir un mismo lenguaje. No existe transformación posible en la medida en que no lleguemos a comprender las raíces de los problemas. Las relaciones entre Teoría y Práctica son, parafraseando a Carretero, relaciones entre tres elementos, dado que debiéramos agregar aquí la manera en que se coordinan ambas. Es en este espacio precisamente donde intentamos abrir una rendija para asomarnos a la complejidad del ejercicio de la docencia en el primer año de práctica, donde el principiante se enfrenta a la necesidad de articular el conocimiento teórico y trazar su camino en el aula.

Encontramos que los novatos investigados no encajan en la descripción de las etapas en la vida profesional de los docentes relevados. En primer lugar, porque están siendo acompañados en su práctica por diferentes actores, lo que hace que ésta no sea solitaria. En segundo lugar, porque las preocupaciones de la etapa de iniciación no están presentes aquí de la forma esperada. Pensábamos encontrarlos angustiados por la disciplina, y pese a que existen problemas de dominio de grupo, no se manifiesta como una de sus preocupaciones centrales.

Al contrastar con el modelo de Leithwood, por ejemplo, encontramos que nuestros novatos son capaces de contribuir al desarrollo didáctico de sus compañeros, lo que aparece recién en la quinta etapa de este teórico, y es efecto de la apertura del aula. Si observamos las etapas de Hargreaves, y por el mismo motivo, no se daría el individualismo ni la práctica en solitaria, sino que en alguna medida, se encontrarían en la etapa tercera, que corresponde al profesional colegiado por cuanto consideran a sus colegas como fuentes de aprendizaje, orientación y apoyo mutuo. 
En otros aspectos se comportan como novatos prototípicos en su primer año de ejercicio: se produce una imitación acrítica de conductas observadas en otros profesores (Marcelo). Hemos argumentado respecto a lo que dimos en llamar "mimesis cegadora", por cuanto la visión del docente experiente que tutorea la práctica incide de forma notoria en los principiantes. De alguna forma, este acompañamiento que reciben minimiza el "reality shock" en este primer año, no obstante, ello no se manifiesta en prácticas innovadoras como resultante de este seguimiento. Tal como lo advierte Messina (2000) la formación docente en América Latina ha quedado atrapada en modelos tradicionales de enseñanza y aprendizaje. Su función queda relegada, entonces, a facilitar la adaptación del principiante a la "gramática de la escuela". Si bien no están solos y logran mayor seguridad en el aula, ello no se traduce en un acercamiento a etapas más plenas del profesionalismo docente en términos de Aguerrrondo. Siguiendo en esta última línea, que es también la de Tedesco, intentamos averiguar la distancia de estos principiantes a la profesionalización. Si el profesionalismo pasa por la autonomía para tomar decisiones y la responsabilidad que se asume frente a las mismas, nuestros practicantes están bastante lejos de ello. En primera instancia, porque en este modelo la autonomía es relativa en la medida en que la presencia de los docentes encargados de tutorearlos es tan fuerte que no les deja espacio para ejercerla. En segunda instancia, porque no asumen las responsabilidades dado que siempre encuentran un chivo expiatorio, generalmente los alumnos, aunque puede variar: la dirección del liceo, el hecho de ser practicantes, el horario de las clases, la falta de tiempo y la disposición del salón.

Nuestros novatos se ven atrapados por una multicausalidad de factores que les impiden un mayor grado de desarrollo profesional, entre las que cabe reseñar las intermediaciones del sistema y el poder de la organización, las prácticas tradicionales de las que han sido objeto, el bajo impacto de la formación inicial, y la saturación atencional. Esta última es producto del régimen intenso del modelo Ce.R.P.; además de las presiones conocidas bajo las que cualquier principiante se halla, en estos centros se añaden las jornadas de tiempo completo, que les dejan poco margen para el estudio, la reflexión y la maduración. De este modo, como no están en condiciones de atender todas las exigencias, se saturan y optan por aquellos aspectos que detectan como prioritarios desde la visión del tutor. Esto último es lógica consecuencia de haber adquirido el oficio de alumno, aunado a la mimesis cegadora. Este comportamiento mimético identificado no significa necesariamente la adopción del modelo docente de referencia, sino simplemente la marca en este primer año de ejercicio profesional, durante el cual están fuertemente guiados por el tutor, que tiene en sus manos el poder de la acreditación final. Al analizar en conjunto la práctica en esta modalidad innovadora, cabe preguntarse hasta qué punto esta fuerte convivencia, que genera lazos afectivos positivos y negativos, no opera como una especie de cortina que impide verse con claridad y objetividad en el espejo que los practicantes son de nosotros mismos.

La apertura del aula es, sin lugar a dudas, una característica de nuestros novatos, dado que se inician en el aula en parejas pedagógicas, compartiendo el grupo y las responsabilidades consiguientes. Al mismo tiempo, se entrenan en un espacio en el que confluyen múltiples miradas sobre la práctica. Los egresados de esta modalidad cuentan con una fortaleza en este sentido, la que independientemente de todos los discursos, han vivenciado en su formación y nos permite afirmar, sin temor a equivocarnos, que salen a los liceos dispuestos a revisar sus prácticas con el aporte de otros actores de la comunidad educativa. 
En cuanto al discurso interrogativo, es algo a repensar desde la institución formadora, puesto que las preguntas en el aula deberían ser objeto de atención central por su función motivadora. El conocimiento científico avanza en torno a preguntas, lo cual es válido no sólo para el investigador, sino también para el alumno que llega a la educación formal con curiosidad y deseos de aprender. Es deber del enseñante alimentar su espíritu inquieto y desarrollar en él la capacidad de autonomía en la búsqueda incesante de respuestas que generen nuevas preguntas.

Precisamente porque buscábamos respuestas, nos encontramos con nuevas preguntas sobre la formación docente desde la visión de Fullan como el peor problema y la mejor solución en educación. Se nos abre así un abanico de interrogantes:

Si la nuestra es una empresa de bajo impacto, ¿la clave no pasará por atender, con un enfoque sistémico, lo que sucede en la socialización profesional para evitar que se lave (washed out $)^{12}$ lo aprendido en la formación de grado?

Si esto fuera así, ¿la política educativa no debería priorizar los programas de formadores de formadores y crear figuras de mentorazgo en los centros educativos que trabajen en forma coordinada con los institutos de formación docente?

¿Los centros de práctica no deberían transformarse en espacios de reflexión colegiada?

¿Qué andamios debiéramos construir para ayudar al novato a pasar a una etapa de profesionalismo pleno? ¿Qué recorridos conceptuales optimizarían las clínicas de Didáctica? ¿Cómo ayudar al novato a obtener el conocimiento declarativo correcto que le permita automatizar algunos procedimientos y así hacer más eficiente su labor?

¿Cómo evitar la mimesis cegadora? ¿Será conveniente evitarla o se tratará de un paso necesario para la construcción del rol docente?

¿Cómo recuperar la real dimensión de la pregunta para el espacio del aula?

Este breve interrogatorio pretende esbozar la problematización de la cuestión, sacándola del campo del discurso declarativo-normativo y partir de un giro empírico que permita pensar en la formación docente como objeto de estudio despojada de históricos reduccionismos ${ }^{13}$.

"La ciencia es una religión corta. Como en las demás religiones, la turba no iniciada cree a pies juntos y son los altos sacerdotes los que vacilan. Hay devotos de los rayos $\mathrm{X}$ y de San Expedito. Para los débiles, dudar es desplomarse; para los fuertes, dudar es creer. Los que suspendidos en el vacío de la duda avanzan sin caer, son los que tienen alas". El escritor español Rafael Barrett poetiza nuestro sentir: desde el doble rol que implica dirigir un centro de formación de profesores e investigar un aspecto tan esencial como la práctica docente, la duda debe ser nuestra fiel compañera. Estamos en un proyecto innovador, que requiere de imaginación e iniciativa para su sustentabilidad ${ }^{14} \mathrm{y}$ en el que creemos firmemente, pero despojados de necedad ideológica. Como lo expresara Paulo Freire: "La educación, en verdad, necesita tanto de formación técnica, científica y profesional como de sueños y utopías". 


\section{Bibliografía y notas}

Aguerrondo, I. 2002. Los desafíos de la política educativa relativos a las reformas de la formación docente. En: El desempeño de maestros en América Latina y Caribe: Nuevas prioridades. Brasilia: 10-12 julio 2002. Disponible en Internet: <http://www.reduc.cl>.

ANEP-CODICEN. 1997. Centros Regionales de Profesores. Documento base. Montevideo, Mimeo.

ANEP-CODICEN. 1998. Nuevas orientaciones de práctica docente para tercer año de los Ce.R.P. Montevideo, Mimeo.

ANEP-CODICEN. 1999. Análisis del perfil socioeconómico de los estudiantes de los Centros Regionales de Profesores. Montevideo, Unidad de Reprodocumentación, CODICEN.

ANEP-CODICEN. 2000. Una visión integral del proceso de reforma educativa en Uruguay 1995-1999. Montevideo, Impresores asociados.

ANEP-MESyFOD. 1996. Docentes de Secundaria a un año del censo de octubre de 1995 La Reforma de la Educación. Documento V. Montevideo, Anep.

ANEP-SECRETARÍA DE CAPACITACIÓN Y PERFECCIONAMIENTO DOCENTE. 2000. Estructura organizacional de los Ce.R.P. Principales resoluciones que la fundamentan. Montevideo, Mimeo.

ANEP-UNESCO-IIPE. 2003. Los docentes uruguayos y los desafíos de la profesionalización. Informe de difusión pública de resultados. Montevideo, Rosgal.

Aparicio, J. 1995. El conocimiento declarativo y procedimental que encierra una disciplina y su influencia sobre el método de enseñanza. En: Tarbiya, Revista de investigación e innovación educativa, 10, p.:23-38.

Barrios, G. et. al. 1993. Planificación y políticas lingüísticas en Uruguay. México. Iztapalapa. Behares, L. (2003) Portugués del Uruguay y Educación Fronteriza. Montevideo, Facultad de Humanidades.

Burbules, N. 1999. El diálogo en la enseñanza. Teoría y práctica. Buenos Aires, Amorrortu.

Careaga, A. 2002. La formación inicial de Profesores de Educación Media en Uruguay en el ámbito de los Centros Regionales de Profesores. Una tensión entre la tradición y la innovación. En: El desempeño de maestros en América Latina y Caribe: Nuevas prioridades: Actas. Brasilia: 10-12 julio 2002.

Carretero, M. 1997. Introducción a la psicología cognitiva. Buenos Aires: Aique.

CIFRA-MEMFOD. 2003. Estudio del impacto de la formación de los egresados de los Centros Regionales de Profesores en la primera etapa de su ciclo profesional. Montevideo, Mimeo.

Davini, M. C. 1995. La formación docente. Un programa de investigaciones. En: Revista IICE, Año IV N 7, pp. 33-46, diciembre de 1995.

De La Torre, S. y Barrios, O. (coordinadores) 2000. Estrategias didácticas innovadoras. Recursos para la formación y el cambio. Barcelona,Octaedro.

Elizaincin, A. (1992) Dialectos en contacto. Español y Portugués en España y América. Montevideo: Arca.

Fullan, M. 1993. Change forces: Probing the depths of Educational Reform. Londres, Palmer. Gabbiani, B. 2000. Escuela, lenguaje y poder. Montevideo, Udelar, F.H.C.E. 
Hargreaves, A. 1996. Profesorado, cultura y postmodernidad. Madrid, Morata.

Huberman, M. 1992. The lives of teachers. Londres, Cassell.

Lortie, D. 1975. Schoolteacher. Chicago, University of Chicago Press.

Mancebo, E. 2003. Políticas de formación docente, profesionalización y equidad. El caso de los Centros Regionales de Profesores en el marco de la reforma educativa uruguaya. Montevideo, Universidad Católica del Uruguay (tesis doctoral en prensa).

Marcelo García, C. 1991. Aprender a enseñar. Un estudio sobre el proceso de socialización de los profesores principiantes. Madrid, CIDE.

Marcelo García, C. 1999. Formación del profesorado para el cambio educativo. $2^{a}$ edición. Barcelona, EUB.

Mercer, N. 1997. La construcción guiada del conocimiento. El habla de profesores y alumnos. Barcelona, Paidós.

Messina, G. 2002. Investigación en o Investigación acerca de la Formación Docente: un estado del arte en los noventa. Santiago de Chile, Universidad de los Lagos. Disponible en Internet: <http://www.reduc.cl >.

Rodríguez Moneo, M. 1999. Conocimiento previo y cambio conceptual. Buenos Aires: Aique. Sautu, R. 2003. Todo es teoría. Objetivos y métodos de investigación. Buenos Aires, Ediciones Lumiere.

Sikes, P. 1985. The life cicle of the teacher. En Ball, S.J y Goodson, I.F. (Eds.) Teacher's lives and careers. Londres, The Falmer Press.

Tedesco, J. C. 1998. Current trends in educational reform. En Education for the TwentyFirst Century, Issues and Prospects, Contributions to the work of the International Commission on Education for the Twenty-First Century, chaired by Jacques Delors. París, Unesco Publishing.

Terhart, E.1987. Formas del saber pedagógico y acción educativa o ¿qué es lo que forma en la formación del profesorado? En: Revista Educación, № 284, Madrid.

Torres, R. M. 1996. Formación docente: clave de la reforma educativa. En: Nuevas formas de aprender y enseñar. Santiago, Unesco- Orealc. Disponible en Internet:

$<$ http://www.reduc.cl >.

Vaillant, D. 2002. Formación de formadores. En: El desempeño de maestros en América Latina y Caribe: Nuevas prioridades. Actas. Brasilia: 10-12 julio 2002.

Vaillant, D.; MARCELO, C. 2000. ¿Quién educará a los educadores? Montevideo, Impresora Editorial.

Wittrock, M. 1990. La investigación en la enseñanza, III. Profesores y alumnos. Barcelona, Paidós.

${ }^{1}$ Nos referimos a las publicaciones: ANEP-CODICEN. 1997. Centros Regionales de Profesores. Documento base. Montevideo: Mimeo.

ANEP-CODICEN. 1998. Nuevas orientaciones de práctica docente para tercer año de los Ce.R.P. Montevideo: Mimeo.

ANEP-CODICEN. 2000. Una visión integral del proceso de reforma educativa en Uruguay 1995-1999. Montevideo: Impresores asociados.

ANEP-MESyFOD. 1996. Docentes de Secundaria a un año del censo de octubre de 1995. La Reforma de la Educación. Documento V. Montevideo: Anep.

ANEP-SECRETARÍA DE CAPACITACIÓN Y PERFECCIONAMIENTO DOCENTE. 2000. Estructura organizacional de los Ce.R.P. Principales resoluciones que la fundamentan. Montevideo: Mimeo. 
${ }^{2}$ El ajuste curricular que se implementa a partir de 2005 mantiene las características y carga horaria total, pero extendiendo la carrera a cuatro años.

${ }^{3}$ Esta especialidad se ofrece únicamente en los Ce.R.P. de Atlántida, Colonia y Salto.

${ }^{4}$ Para el año lectivo 2004, por primera vez se crearon dos grupos de cuarto año, los cuales comenzaron a funcionar en el turno intermedio.

${ }^{5}$ Los barrios Mandubí y La Virgencita, altamente poblados, se encuentran a $5 \mathrm{kms}$. de la ciudad por la ruta 5 , mientras que el Liceo 4 se encuentra también a $5 \mathrm{kms}$. de Rivera, pero por la Avenida Italia. Insólitamente, no existe aún una vía de tránsito interna que una estos barrios, por lo que no hay más alternativa que ir hasta la ciudad y de allí tomar el otro camino.

${ }^{6}$ Los tutores y profesores de apoyo realizan un acompañamiento de cada practicante, asistiendo a sus clases, aportando críticas y sugerencias, guiándolo en la selección de recursos, en los contenidos a profundizar, indicando bibliografía, e incluso continentando emocional y afectivamente su proceso.

${ }^{7}$ Término popularizado por el holandés Simon Veenman (1984), apud. Marcelo (1999).

${ }^{8}$ Diglosia es una situación de uso de dos lenguas en la cual existe una distribución de funciones para cada una de ellas. El español es utilizado como variedad alta para todos los fines formales (educación, religión, sistema judicial, administración y prensa) y los D.P.U. (dialectos portugueses en el Uruguay) son utilizados exclusivamente en la comunicación intrafamiliar y para la comunicación espontánea entre los miembros de un mismo grupo social.

${ }^{9}$ Esta afirmación tiene su fundamento en el estudio: ANEP-CODICEN. 1999 Análisis del perfil socioeconómico de los estudiantes de los Centros Regionales de Profesores. Montevideo, Unidad de Reprodocumentación, CODICEN.

${ }^{10}$ Se denominan rasgos suprasegmentales en Gramática a los elementos que acompañan lo verbal como la entonación, la curva melódica y los esquemas acentuales.

${ }^{11}$ En De la Torre y Barrios (2000).

12 Zeichner y Tabachnick (1981) apud Davini (1995).

${ }^{13}$ En términos de Davini(1995).

${ }^{14}$ Si bien la sustentabilidad de una innovación debe ser académica, administrativa y económica, nos podemos responsabilizar por la primera de ellas, puesto que las otras dos dependen de decisiones de política educativa, competencia de la autoridad del ente.

*Master en Educación, Universidad ORT. Diploma en Gestión Educativa, Universidad ORT. Diploma en Educación, Universidad ORT. Diplome de Hautes Études Supérieures option Littérature Francaise, Alliance Francaise de IÚruguay. Certificado de actualización en Gestión Educativa, Universidad ORT. Profesora de Literatura e Idioma Español, Instituto de Profesores Artigas. 